\title{
Organotypic 3D Culture in Nanoscaffold Microwells Supports Salivary Gland Stem-Cell-Based Organization
}

\author{
Hyun-Soo Shin, ${ }^{\dagger}$ Hye Jin Hong, ${ }^{\ddagger}$ Won-Gun Koh, ${ }^{*}, \S_{\odot}$ and Jae-Yol Lim ${ }^{*} \dagger, \S_{\odot}$
}

${ }^{\dagger}$ Department of Otorhinolaryngology, Yonsei University College of Medicine, and ${ }^{\ddagger}$ Department of Chemical and Biomolecular Engineering, Yonsei University, Seoul 03722, Republic of Korea

\section{Supporting Information}

ABSTRACT: The self-organizing properties of stem cells have been exploited to generate organoids, organ-specific, cell-containing, three-dimensional (3D) structures. The present study aimed to introduce a novel bioengineering technique for driving the effective organization of adult tissue stem cells via niche-independent $3 \mathrm{D}$ microwell culture. Microwells were fabricated by photopatterning poly(ethylene glycol) hydrogel in the presence of an electrospun polycaprolactone nanofibrous scaffold. Human single clonal salivary gland stem cells (SGSCs) were cultured in nanofibrous microwells through two simple steps, priming and differentiation. Before the induction of $3 \mathrm{D}$ organization, single clonal SGSCs were preconditioned to aggregate to form 3D spheroids in different matrices, such as Matrigel, floating dish, and microwells. Expression of salivary stem cell markers and pluripotency markers was greater in 3D spheroid cultures than in 2D plastic culture. Lobular structures were organized by changing media, and those in microwells exhibited higher salivary acinar, ductal, and tight junction marker levels and decreased stem-cell marker levels relative to other $3 \mathrm{D}$ cultures. Furthermore, higher $\alpha$-amylase secretion and intracellular calcium levels were observed in the presence of adrenergic or cholinergic agonists, respectively, along with an increased intracellular $\mathrm{pH}$, suggesting more functional salivary organoid formation. These microwell-driven organoids also engrafted successfully into nonobese diabetic/severe combined immunodeficient (NOD/SCID) mice. Our results showed that microwellcultured SGSCs organize into salivary structures and that this biomimetic 3D culture technique can promote effective generation of niche-independent single stem-cell-based 3D organoids.

KEYWORDS: salivary glands, multipotent stem cells, differentiation, three-dimensional culture

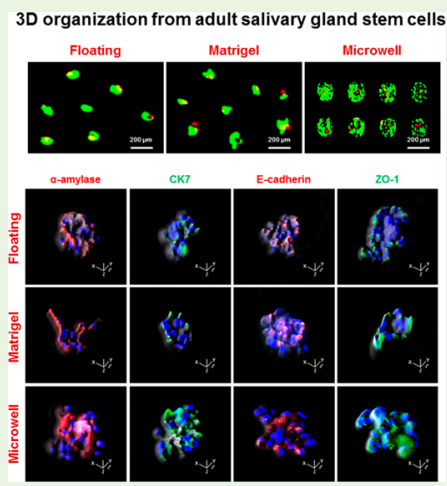

\section{INTRODUCTION}

Scientific advances in 3D culture technology have afforded researchers the opportunity to address specific questions concerning complex in vivo organs in a dish. Three-dimensional (3D) culture systems encompass various organotypic cultures, such as $3 \mathrm{D}$ cell aggregates, tissue explants or slices, or 3D spheroid cultures. ${ }^{1}$ In comparison with $2 \mathrm{D}$ monolayer cultures, in which the maintenance of in vivo-like phenotype and function are hampered by a lack of cell-cell and cell-matrix interactions, $3 \mathrm{D}$ culture systems are able to mimic the in vivo microenvironment and to reproduce physiologically relevant cell-microenvironment interactions. ${ }^{2}$ However, there are still areas for improving 3D organotypic cultures: 3D cell aggregates often fail to show relevant tissue organization, and tissue explants or slices possess limited availability, because of the rapid loss of transient cell organization and the difficulty of long-term maintenance. Although 3D spheroid cultures show promise for sustained culture, they are often unable to assemble into complex structures in the absence of multipotent cells with the capacity of self-renewal and differentiation. ${ }^{2}$

In recent years, it was proposed that stem cell-based organoids can reduce a gap in the capabilities of existing 3D culture systems. Organoids are defined as a 3D structure consisting of organ-specific cell types that is self-organized from stem cells. ${ }^{3}$
There are two types of stem cells available for the generation of organoids: (1) pluripotent stem cells (PSCs), which include embryonic stem cells or induced pluripotent stem cells, and (2) organ-specific adult stem cells (ASCs). ${ }^{3}$ PSC-based organoid generation mainly replicates the developmental process and organogenesis, in which PSCs are induced to differentiate into specific lineages in the presence of specific transcription factors. $^{4-7}$ However, in the process of ASC-based organoid formation, stem cell niche components are harnessed, which encompass a number of biochemical and biophysical signals. ${ }^{8-10}$ Although the organoid-generating processes using these essential niche factors are useful, niche factor-independent organization from ASCs appears to have been achieved by engineering stem cells under microenvironment-mimetic culture conditions. ${ }^{2}$

We previously isolated salivary gland-derived stem cells (SGSCs) by subfractionation culture from mice and humans and found that these cells retained multipotent tissue stem cell features. $^{11,12}$ Given that ACSs self-organize to form tissuespecific cells, in this study, we aimed to induce salivary

Received: August 1, 2018

Accepted: October 17, 2018

Published: October 17, 2018 
organization from single clonal SGSCs without exogenous niche factors under $3 \mathrm{D}$ culture conditions. To this purpose, we employed our nanofibrous microwell culture, with which we had previously assembled a robust acinar-like structure based on $3 \mathrm{D}$ spheroid culture of human parotid epithelial cells (hPECs). ${ }^{13}$ After we observed that it could provide an in vivo- like microenvironment and promote the formation of functional acini to secrete salivary proteins from hPECs, we were prompted to evaluate subsequent steps through which to organize more complex and larger functional tissues from multipotent SGSCs in this study. To investigate the organization efficacy of SGSCs, we explored the potency of the formation of salivary organoids in terms of cellular morphology, structure, and function and compared it with 2D plastic and traditional 3D culture systems, including Matrigel and floating cultures. The results presented herein confirm that single clonal SGSCs can differentiate and assemble into salivary organoids under biomimetic 3D culture conditions without niche factors.

\section{MATERIALS AND METHODS}

More detailed information about the materials used in the experiments is given in Tables $\mathrm{S} 1-\mathrm{S} 4$.

Cultivation of Human Single Clonal SGSCs and their Multipotent Stem Cell Features. We previously established single SGSC clones isolated from human parotid glands using a modified subfractionation culture method, which has been shown to be effective for the isolation of highly homogeneous clonal stem cells. ${ }^{12}$ For this experiment, cryopreserved single clonal SGSCs stored in liquid nitrogen vapor were thawed and recultured in DMEM (Gibco) supplemented with 10\% FBS (Gibco) and $1 \%$ penicillin/streptomycin (Gibco). The culture-expanded SGSCs confirmed to have multipotent stem cell characteristics were used for experiments. All experimental procedures were performed in accordance with the guidelines and regulations of the Institutional Review Board with informed consent acquisition (2015-10-011-002).

Preparation of Hydrogel Micropatterned Nanofibrous Microwells. Nanofibrous scaffolds were prepared by a conventional electrospinning process as previously described. ${ }^{13,14}$ Briefly, electrospinning solution was prepared by dissolving polycaprolactone (PCL; MW: 80,000$)$ in 2, 2, 2-trifluoroethanol to form a $20 \% \mathrm{w} / \mathrm{w}$ solution. The PCL solution was electrospun (NanoNC) for $20 \mathrm{~min}$ through a $23 \mathrm{G}$ needle with a $6.5 \mathrm{kV}$ positive voltage at a constant flow rate of 0.5 $\mathrm{mL} / \mathrm{h}$. The PCL nanofibrous sheets were collected on cover glasses wrapped with clean aluminum foil during the electrospinning process, and the sheets were further treated with oxygen plasma (Femto Science) for $2 \mathrm{~min}$ to increase the hydrophilicity of PCL fibers.

The electrospun nanofiber scaffolds were incorporated into hydrogel micropatterns via photolithography. First, hydrogel precursor solution $(200 \mu \mathrm{L})$ consisting of 2-hydroxy-2-methylpropiophenone $(2 \% \mathrm{v} / \mathrm{v})$ and poly(ethylene glycol)-diacrylate (50\% w/w) (PEG-DA; MW575) in deionized water was dropped onto the electrospun fibers. After the precursor solution was completely spread out over the sheet, a photomask representing square microarray patterns $(200 \times 200 \mu \mathrm{m})$ was placed on the nanofiber sheet. Exposure of ultraviolet light (365 $\mathrm{nm}$, EFOS Ultracure $100 \mathrm{ss}$ Plus, UV spot lamp) for $1 \mathrm{~s}$ was sufficient to induce free-radical cross-linking polymerization. After cross-linking, unreacted precursor solution was washed away with water. For cell studies, scaffolds were sterilized in $70 \% \mathrm{v} / \mathrm{v}$ ethanol solution for $30 \mathrm{~min}$, and then washed three times in phosphate-buffered saline (PBS) to remove the ethanol. The resultant hydrogel micropattern-incorporated fibrous sheet was obtained as an array of microwells formed from the nanofiber bottom and hydrogel wall.

3D Priming Cultures of hSGSCs in Different Culture Matrices. Before the induction of 3D organization, cells were preconditioned to aggregate to form $3 \mathrm{D}$ spheroids in different matrices. The validated single clonal cells at passage 3 were then seeded in 6-well plates that had been precovered with Pluronic F-127 (Sigma), Matrigel (Corning), or PCL microwells at a density of $2 \times 10^{5}$ cells/well, after which the cells were cultured for 7 days in DMEM (Gibco) supplemented with $10 \%$ FBS (Gibco) and 1\% 1\% penicillin/streptomycin (Gibco). The 2D plastic culture was used as a 2D control.

Generation of 3D Organoids in Different Culture Matrices. At 3 days after priming cultures, the initiation of lineage commitments was induced by organoid culture. Briefly, single clonal SGSCs at passage 3 were cultured on 6-well plates precovered with Pluronic F-127, Matrigel, or PCL microwells at a density of $2 \times 10^{5}$ cells/well, after which they were cultured in serum-free hepato-STIM medium (Corning) supplemented with recombinant EGF (R\&D system), 2 $\mathrm{mM}$ L-glutamine, and $1 \%$ penicillin/streptomycin at $37^{\circ} \mathrm{C}$ for 3 days.

Evaluation of Stem-Cell Properties. Spheroid-Forming Activities. Cell morphology was analyzed at 1, 3, 5, and 7 days after priming culture by an inverted phase-contrast microscope (Olympus), and the images were obtained with a digital camera. To evaluate the sphereforming ability on each substrate, the diameter of the spheroids was measured 3 days after culture and then normalized by the total number of spheroids. Spheroid-forming efficacy was further determined by the measurement of the average number of spheroids per plate at 3 days after plating the same number of cells.

Cell Viability. To assess the cell viability, we utilized a LIVE/DEAD assay kit (Thermo Scientific) in accordance with the manufacturer's instructions. Briefly, SGSCs were seeded on the substrates in 6-well plates at a density of $3 \times 10^{4}$ cells/well. At 3 days after culture, cell viability was measured by exposure of cells to LIVE/DEAD ( $2 \mathrm{mM}$ EthD-green, for live cells, and $4 \mathrm{mM}$ Calcein AM-red, for dead cells) for $30 \mathrm{~min}$ at $25^{\circ} \mathrm{C}$. The cells were then visualized under an Axiovert 200 fluorescence microscope (Carl Zeiss). We further performed a Trypan blue dye (Thermo Scientific) exclusion assay. At 1, 3, 5, and 7 days, the cells were washed with $1 \times$ PBS, detached by the addition of $0.05 \%$ Trypsin-EDTA, neutralized with cell culture media, and collected in tubes. Next, cells were centrifuged at $127 \times g$ for $5 \mathrm{~min}$, after which the supernatant was discarded, the pellets were resuspended in Trypan blue dye in media for $1 \mathrm{~min}$, and the viable cells were counted using a hemocytometer. The cell viability percentage was determined based on the viable cell count divided by the total cell count. A minimum of least three independent experiments were performed.

Evaluation of Salivary and Stem-Cell-Related Gene Expression. Transcript levels were determined by real-time PCR (RT-PCR) using an ABI PRISM sequence detection system with SYBR Green I as a double-stranded DNA-specific dye in accordance with the manufacturer's instructions (Applied Biosystems). The PCR was conducted with $1 \mu \mathrm{M}$ cDNA, $10 \mu \mathrm{M}$ SYBR Green PCR master mix (Thermo Scientific), and $10 \mathrm{pM}$ sense and antisense primers specific to each gene. The relative expression levels were normalized to the housekeeping gene GAPDH, and a minimum of three independent experiments were conducted in triplicate for each sample.

Scanning Electron Microscopy for Evaluation of Cellular Morphology. The morphology of cells in the Pluronic F-127, Matrigel, or PCL microwells was also observed by scanning electron microscopy (SEM). Samples were fixed in $4 \%$ paraformaldehyde (30 $\mathrm{min}$ at room temperature) and then rinsed in $1 \times$ PBS. The specimens were dehydrated using different concentrations of ethanol and dried in a critical point dryer (HCP-2, Hitachi Koki Co., Ltd.). The samples were then sputter-coated with platinum at $15 \mathrm{~mA}$ for $60 \mathrm{~s}$ prior to SEM imaging. SEM images were obtained with field-emission scanning electron microscopy (JEOL-7001F) at the acceleration voltage of 10 $\mathrm{kV}$.

Immunofluorescent Staining for Evaluation of Composition of Salivary Organoids. The cells were washed twice with PBS, fixed with $4 \%$ paraformaldehyde at $25^{\circ} \mathrm{C}$ for $20 \mathrm{~min}$, and then washed in PBS containing $0.05 \%$ Tween 20 . Nonspecific binding was blocked by incubation of the cells with PBS containing $1 \%$ bovine serum albumin (BSA). The cells were then incubated with primary antibodies to anti$\alpha$-amylase, anti-Cytokeratin 7 and anti-ZO-1 (Santa Cruz Biotechnology), and anti-E-cadherin (BD Bioscience) in a moist chamber overnight at $4{ }^{\circ} \mathrm{C}$. After washing in PBS, the cells were incubated with goat antimouse IgG-Alexa-488-conjugated and goat antirabbit IgGAlexa-555 secondary antibodies (Invitrogen) for $2 \mathrm{~h}$ in the dark at 25 ${ }^{\circ} \mathrm{C}$. Next, 4',6-diamidino-2-phenylindole, dihydrochloride (DAPI; 
A

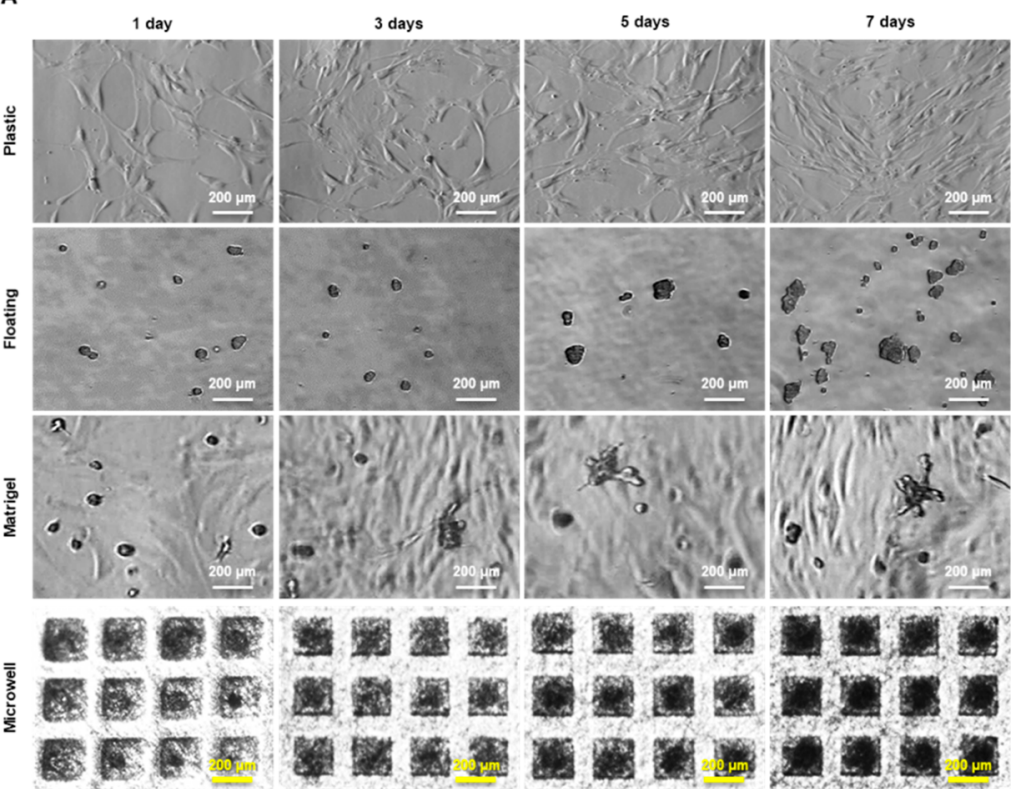

D
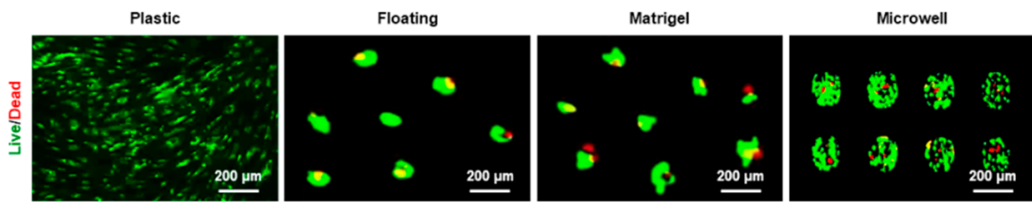

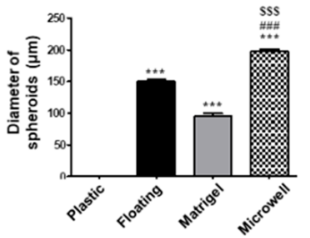

C
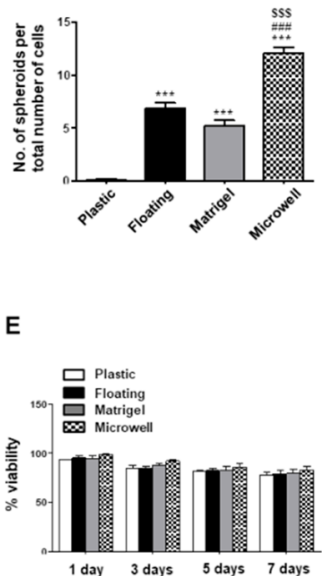

Figure 1. 3D spheroid formation and viability of SGSCs under a priming (spheroid) culture. (A) Floating, Matrigel, and microwell scaffolds induced SGSCs to aggregate and assemble into 3D spheroids in a time-dependent manner. Scale bars $=200 \mu \mathrm{m}$. (B) Diameter of spheroids was measured after culture for 3 days, and the values were normalized to the total number of spheroids. The data from five independent experiments were analyzed and presented as a mean \pm standard errors of the mean $(n=5)$. One-way ANOVA, Tukey's post hoc test. *, compared with plastic; \#, compared with

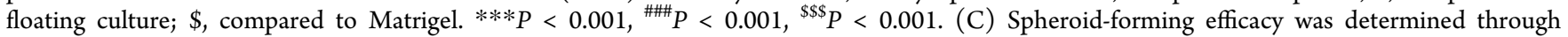
measurement of the average number of spheroids per plate after plating the same number of cells. The data from five independent experiments were analyzed and presented as the mean \pm standard errors of the mean $(n=5)$. One-way ANOVA, Tukey's post hoc test. *, compared with plastic; \#, compared with floating culture; $\$$, compared with Matrigel. $* * * P<0.001,{ }^{\# \# \#} P<0.001,{ }^{\$ \$ \$} P<0.001$. (D) A representative LIVE/DEAD fluorescence image of hPECs spheroids after culture for 5 days. (E) Cell viability percentage (viable cell count/total cell count) was measured at $1,3,5$, and 7 days by the Trypan blue dye exclusion technique. The data from three independent experiments were analyzed and presented as the mean \pm standard errors of the mean $(n=3)$.

Thermo Scientific) was added for 3-5 min to stain the cell nuclei. All experiments included a slide with no primary antibody as a negative control. After mounting, cells were viewed by using a confocal laser scanning microscope (Olympus FV1000, Olympus, Tokyo, Japan).

Evaluation of Changes in Protein Expression. Samples were isolated from lysates $(30 \mu \mathrm{g})$, mixed in reducing buffer, boiled, resolved on SDS-PAGE gels, and transferred to a PVDF membrane by electroblotting. The blot was incubated overnight at $4{ }^{\circ} \mathrm{C}$ in a blocking solution with primary antibodies to the following antigens: $\alpha$-amylase, AQP5, CK7, CK18, LGR5 and $\beta$-actin (Santa Cruz Biotechnology), CD90, KRT5, NANOG, OCT4, and SOX2 (Abcam). The blots were washed with $0.1 \%$ Tween 20 in $1 \times$ PBS, incubated with horseradish peroxidase-conjugated secondary antibodies that corresponded to each primary antibody, and then subjected to enhanced chemiluminescence detection (GE Healthcare Life Science). The protein band intensities were quantified in three independent experiments, and the relative ratio to the reference protein $\beta$-actin was calculated.

Functional Assessment of Salivary Organoids. Amylase Activity. During the differentiation of SGSCs into SG epithelial cells, changes in the function of SGSCs were evaluated by amylase activity. The SGSCs were seeded on the substrates at $3 \times 10^{4}$ cells/well, and then cultured until the appropriate spheroid formation was reached. Fresh medium was added every 3 days. The SGSCs in 2D monolayers and 3D spheroids were stimulated with epinephrine (Sigma, Epi, 10 $\mu \mathrm{M}$ ) or isoproterenol (Sigma, ISO, $1 \mu \mathrm{M}$ ) in Hepato-STIM culture medium (Corning) for $45 \mathrm{~min}$ before measurement. The amylase activity was measured from media collected at 5 days using an $\alpha$ amylase activity assay kit (Abcam) with 2-chloro-p-nitrophenol linked to maltotriose as the chromogenic substrate, in accordance with the manufacturer's instructions. The $\alpha$-amylase activity of individual samples was directly proportional to increases in absorbance at 405 $\mathrm{nm}$ using a standard laboratory plate reader. Absorbance (OD) was measured in triplicate from three independent experiments, and data were normalized to the total number of counted cells. The negative control was culture medium without cells.

Western Blot Analysis: Amylase in Supernatant. Supernatant was centrifuged to remove cell debris and added to StrataClean Resin (Stratagene). The resin-bound proteins were pelleted by centrifugation and mixed in reducing buffer, boiled, resolved on 10\% SDS-PAGE gels, and transferred to a polyvinylidene difluoride membrane by electroblotting. The blot was incubated overnight at $4{ }^{\circ} \mathrm{C}$ in blocking solution with primary antibodies directed toward the $\alpha$-amylase and $\beta$-actin antigens (Santa Cruz Biotechnology). After the blots were washed with $0.1 \%$ Tween 20 in $1 \times$ PBS, they were incubated with horseradish peroxidase-conjugated secondary antibodies that corresponded to each primary antibody and then subjected to enhanced chemiluminescence detection (GE Healthcare Life Science).

Measurement of Intracellular $\mathrm{CaCl}_{2}\left(\left[\mathrm{Ca}^{2+}\right]_{i}\right)$. The measurement of the intracellular calcium concentrations $\left(\left[\mathrm{Ca}^{2+}\right]_{i}\right)$ in spheroids in the same culture was conducted using the calcium-sensitive fluorescence 

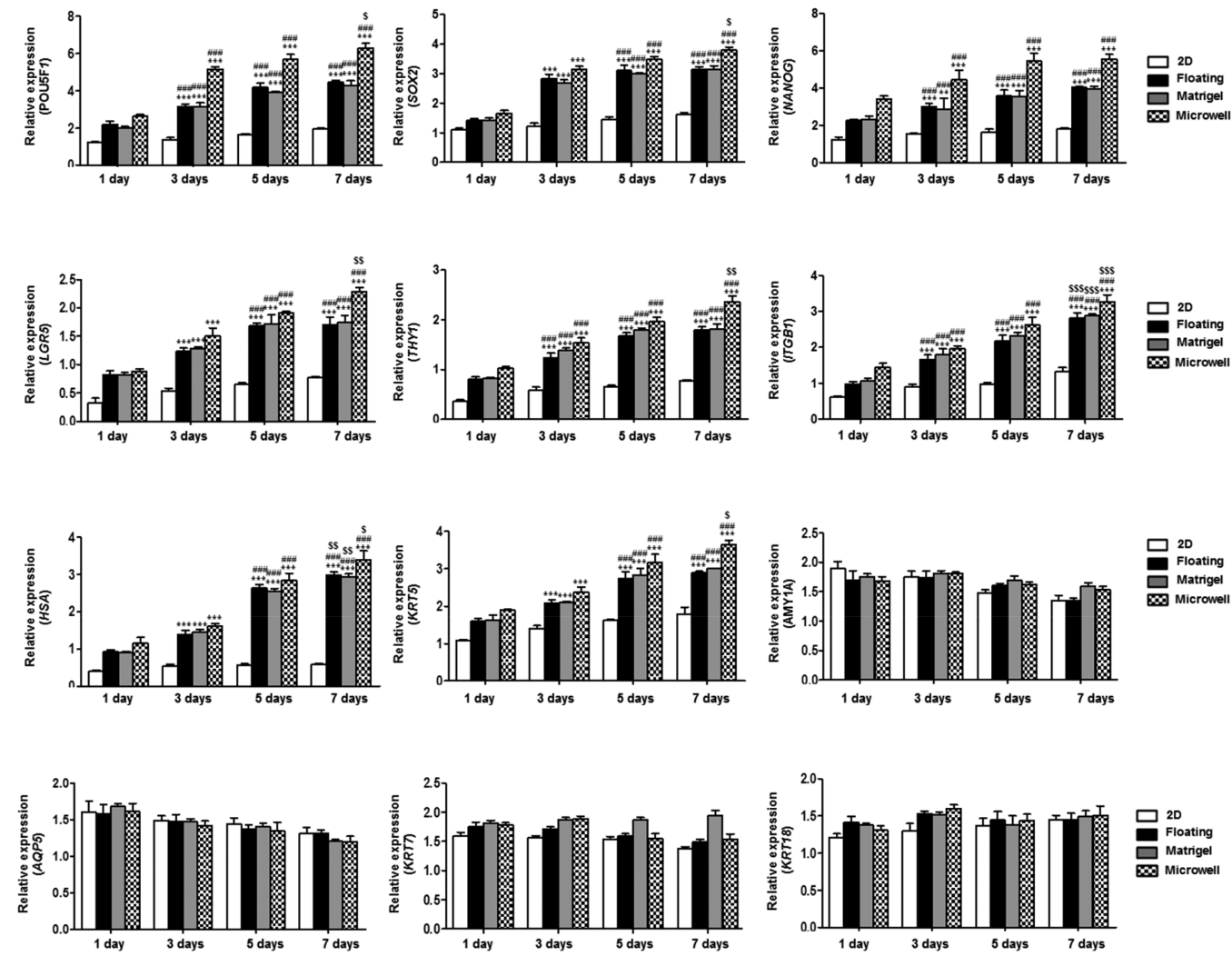

Figure 2. The effect of 3D spheroid culture on gene transcription of SGSCs in floating, Matrigel, and microwell cultures. The mRNA levels of pluripotent stem cell markers (POUSF1, SOX2, and NANOG), salivary stem/progenitor cell markers (LGR5, THY1, ITGB1, HAS, and KRT5), salivary acinar markers (AMY1A and AQP5), and ductal cell markers (KRT7 and KRT18) were tested by real-time PCR, and the expression levels relative to GAPDH were calculated in triplicate after 1,3,5, and 7 days of culture. The data from three independent experiments were analyzed and presented as the mean \pm standard errors of the mean $(n=9)$. Two-way ANOVA, Bonferroni posthoc test: ${ }^{*}$ compared with 1-day group; ${ }^{\#}$ compared with 3-day group; ${ }^{\$}$ compared with 5-day group. ${ }^{*} P<0.05,{ }^{* *} P<0.01,{ }^{* * *} P<0.001,{ }^{\#} P<0.05,{ }^{\# \#} P<0.01,{ }^{\# \# \# P} P<0.001,{ }^{\$} P<0.05,{ }^{\$ \$} P<0.01,{ }^{\$ \$} P<0.001$.

indicator Fluo-4AM (Molecular Probes), in accordance with the manufacturer's instructions. The $3 \mathrm{D}$ spheroids were incubated with Fluo-4AM (Sigma, $10 \mu \mathrm{M}$ ) in Hepato-STIM for $1 \mathrm{~h}$ at $37^{\circ} \mathrm{C}$. The spheroids were then washed twice with PBS, and the measurements were recorded without calcium. To stimulate $\mathrm{Ca}^{2+}$ influx, ATP (adenosine triphosphate, $100 \mu \mathrm{M})$, thapsigargin $(1 \mu \mathrm{M})$, or carbachol $(10 \mu \mathrm{M})$ (Sigma) were added to the media. Fluo-4AM signals were recorded using live cell imaging and confocal microscopy (Olympus), and fluorescence intensities at baseline and poststimulation were measured from three independent experiments and compared among groups.

Measurement of Intracellular $\mathrm{pH}\left(\mathrm{Cl}^{-} / \mathrm{HCO}_{3}{ }^{-}\right.$Exchange: $\mathrm{CBE}$ Activity) for Assessment of Ductal Function. Changes in intracellular $\mathrm{pH}$ were measured by stimulating the $\mathrm{pH}$-sensitive indicator $2^{\prime}, 7^{\prime}$ bis(2-carboxyethyl)-5(6)-carboxyfluorescein)-AM (Sigma, BCECF) at dual excitation wavelengths of 440 and $495 \mathrm{~nm}$ and recording the spectra at an emission wavelength of $530 \mathrm{~nm}$. The 3D spheroids were incubated with BCECF-AM $(6 \mu \mathrm{M})$ in physiological salt solution (PSS) for $45 \mathrm{~min}$ at $25^{\circ} \mathrm{C}$. After the fluorescence was stabilized, the 3D spheroids were washed with PSS for at least $5 \mathrm{~min}$ before the intracellular $\mathrm{pH}$ was measured at $37^{\circ} \mathrm{C}$. $\mathrm{CBE}\left(\mathrm{Cl}^{-} / \mathrm{HCO}_{3}^{-}\right)$activity was measured through the incubation of the cells with $\mathrm{CO}_{2}$-saturated $\mathrm{HCO}_{3}{ }^{-}$-buffered media to acidify the cytoplasm and was initiated by washing the cells with $\mathrm{Cl}^{-}$-free $\mathrm{HCO}_{3}^{-}$-buffered media. BCECF-AM signals were recorded using live cell imaging and a confocal microscope (Olympus), and fluorescence intensity at baseline $\left(\mathrm{Cl}^{-}\right.$-free $\left.\mathrm{HCO}_{3}{ }^{-}\right)$ and poststimulation $\left(\mathrm{Cl}^{-} / \mathrm{HCO}_{3}^{-}\right)$were measured from three independent experiments and compared among groups.

In Vivo Evaluation: Salivary-Organoid Transplant Model. Animal Experiments. Female mice (NOD/SCID) weighing 18-20 g that were 9 weeks old were purchased from the Research Model Producing Center (Orient Bio). Procedures and maintenance were performed in accordance with institutional guidelines approved by the Animal Ethics Committee of Yonsei University Hospital (Permit Number: 2017-0092). The mice were inhalation anesthetized by $2.5 \%$ isoflurane and $97.5 \% \mathrm{O}_{2}$. As a carrier of organoid transplant, a collagen hydrogel (mixed 1:1 of collagen-methyltetrazine and collagen-transcyclooctene) was used. After organoid culture of SGSCs in Microwells, the $3 \mathrm{D}$ assembled organoids were mixed with a collagen hydrogel. Then, the organoids-containing hydrogels were injected into both submandibular glands of the mice via an insulin syringe with a 29-gauge needle.

Histological Examination and Immunofluorescence Staining. At 14 days after the transplant of salivary organoids, the submandibular glands were extirpated. The sections ( 4 um thickness) of formalin-fixed, paraffin-embedded tissues were cut and stained with hematoxylin and eosin (H\&E). For immunofluorescence staining of salivary gland tissues, paraffin sections were dewaxed in xylene for $30 \mathrm{~min}$, after which antigen retrieval was performed by boiling in $10 \mathrm{mM}$ sodium citrate for $2 \mathrm{~min}$. The tissue slices were then incubated with a primary antibody (anti-AQP5 antibody; Alomone Lab, anti-CK18 antibody: Santa Cruz Biotechnology) overnight at $4{ }^{\circ} \mathrm{C}$, and next they were incubated with a secondary antibody, an Alexa-conjugated anti-IgG antibody, for $1 \mathrm{~h}$ at room temperature with 4',6-diamidino-2-phenylindole (DAPI). After mounting, the cells were viewed under a confocal laser scanning microscope (LSM 780, Carl Zeiss).

Statistical Analysis. Statistical analysis was conducted using the GraphPad Prism 5 package (GraphPad). The Mann-Whitney test was used to determine differences between groups. One-way ANOVA followed by Tukey's post hoc test, and two-way ANOVA followed by the Bonferroni post hoc test were used to compare values among groups. Linear regression was applied to evaluate correlations between 
parameters. A value of $P<0.05$ was considered to indicate statistical significance.

\section{RESULTS}

Single Clonal SGSCs Expand and Aggregate to form 3D spheroids When Cultured under 3D Priming Culture Conditions. For SGSC-based organization, we developed a simple two-step process of priming and differentiation of SGSCs in microwells. First, we cultivated and expanded SGSCs with growth medium for 3 days under 2D or 3D culture conditions. The cells cultured as monolayers on plastic dishes did not show morphological changes during culture, whereas those cultured in floating dishes, Matrigel, or microwells aggregated and assembled to form 3D spheroids over time (Figure 1A). To assess the efficiency of sphere-formation, we measured the average diameters and numbers of spheroids after 3 days of culture. By 3D priming culture in microwells, we could obtain more spheroids with a larger diameter than culture in floating or Matrigel cultures (Figure 1B, C). We then performed live/dead and trypan blue assays to determine the effects of the culture on viability of SGSCs cultured in floating culture, Matrigel, or microwells at 1, 3, 5, and 7 days after culture. On the live/dead assay images, green and red fluorescence indicated live and dead cells, respectively. Although a few dead cells were observed in the middle of the spheroids during culture, there were no significant differences in cell death among groups (Figure 1D). The cell viability percentage, determined by the trypan blue exclusion assay in which dead cells were counted to measure the percentage of live cells, was preserved in each group during the induction of spheroid formation (Figure 1E). Collectively, the SGSCs more efficiently assembled as spheroids in microwells relative to those cultured in a floating culture or Matrigel.

Stem Cell Properties Are Primed in 3D Spheroid Culture with Growth Medium. To examine the marker expression of spheroidal SGSCs, we evaluated the mRNA expression of genes, including pluripotent stem cell markers (POU5F1, SOX2, and NANOG), salivary stem/progenitor cell markers (LGR5, THY1, ITGB1, HAS, and KRT5), salivary acinar markers (AMY1A and AQP5), and ductal cell markers (KRT7 and KRT18), was evaluated by quantitative-PCR for up to 7 days. As expected, POU5F1, NANOG, LGR5, THY1, ITGB1, HAS, and KRT5 mRNA showed a time-dependent increase, which indicated the priming of function in SGSCs. Conversely, $A M Y, A Q P 5, K R T 7$, and KRT18 mRNA expression was significantly downregulated for up to 7 days (Figure 2).

Primed SGSCs Assemble into Acinar and Ductal Organoids upon Induction of Differentiation. We next induced salivary epithelial differentiation from primed SGSCs with differentiation medium (Hepato-STIM) under 2D or 3D culture conditions. Upon differentiation induction, SGSCs cultured under 3D culture conditions were changed to form 3D spheroidal structures (Figure 3A). To examine the cellular composition in 3D-organized structures, we conducted immunofluorescence study, and the results showed that the 3D lobular structures indicated the remarkable expressions of salivary acinar $(\alpha$-amylase), ductal (CK7), adhesion protein, Ecadherin, and the tight junction protein $\mathrm{ZO}-1$ (Figure $3 \mathrm{~B}$ ). To assess the distribution of salivary or structural proteins, we observed projection images of the $\mathrm{xz}$ axis by a confocal $\mathrm{z}$-stack (Figure 3C). The z-stack images demonstrated that the salivary or structural proteins were distributed throughout the 3D lobular structures. Collectively, the 3D structures retained the salivary epithelial cell components, including predominantly
A

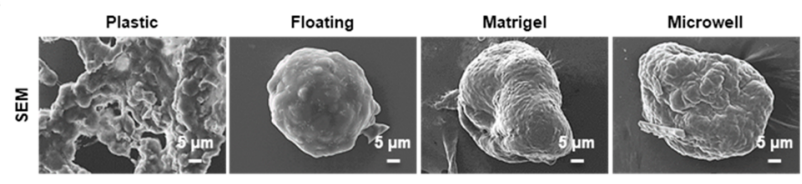

B

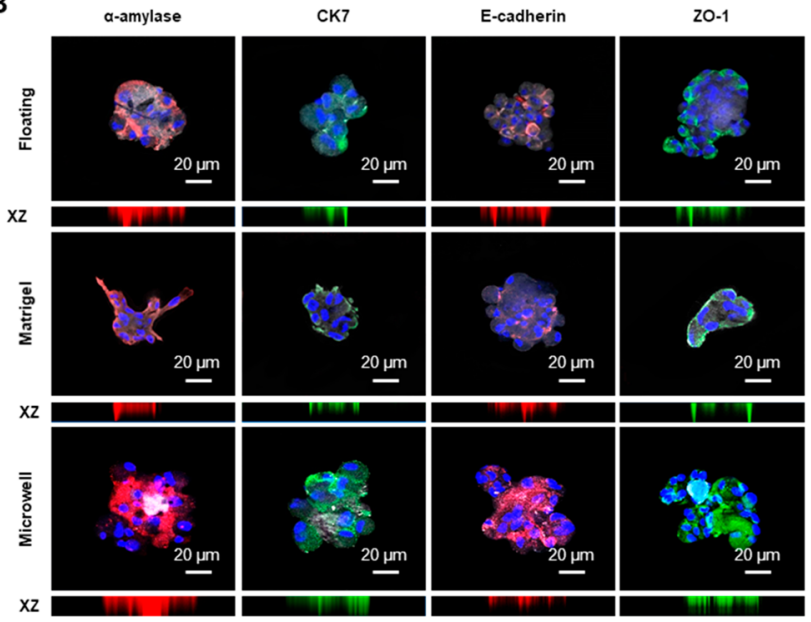

C

a-amylase
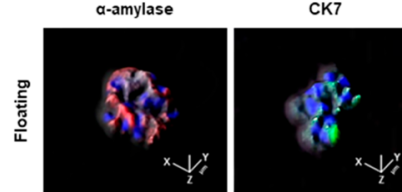

E-cadherin

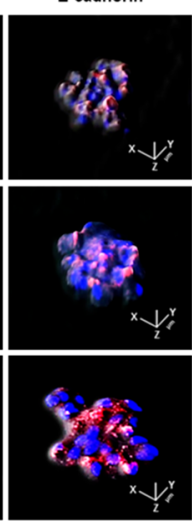

ZO-1

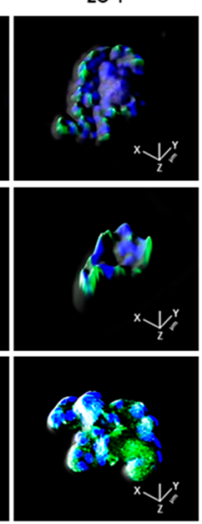

Figure 3. Changes in the structures and phenotypes of SGSCs upon 3D organization induction. (A) Representative scanning electron microscopy image of 3D spheroidal structures upon differentiation induction of SGSCs in the floating, matrigel, and microwell cultures. Scale bars $=5$ $\mu \mathrm{m}$. (B) Immunofluorescence staining for salivary cellular markers in 3D organoids. Salivary acinar markers, $\alpha$-amylase (red), ductal marker, CK7 (green), adherence protein, E-cadherin (red), and the tight junction (TJ) protein ZO-1 (green). The expression levels of $\alpha$ amylase, CK7, E-cadherin, and ZO-1 were enriched in 3D spheroid cultures. Nucleic acids were stained with DAPI (blue). The scale bars represent $100 \mu \mathrm{m}$ in 3D spheroid cultures. Confocal z-stack images with $\mathrm{xz}$ axis show the even distribution of all salivary epithelial markers in 3D spheroids. (C) Salivary structural proteins were uniformly observed in $3 \mathrm{D}$ spherical structures.

acinar and ductal cells, which was suggestive of the formulation of salivary epithelial organoids.

Organization Efficacy of SGSCs is Enhanced in 3D Microwell Culture. To further examine the phenotypic changes in stem cells or epithelial marker expression, we compared the mRNA levels of salivary acinar cell markers (AMY1A and AQP5), salivary ductal cell markers (KRT7, KRT18, NHE1, and SLC26C), intercellular adherence protein $(C D H 1)$, tight junction protein (TJP), and stem cell-related markers (POUSF1, NANOG, LGR5, THY1, ITGB1, HAS, and KRT5) among 2D culture and 3D spheroid cultures after 
A
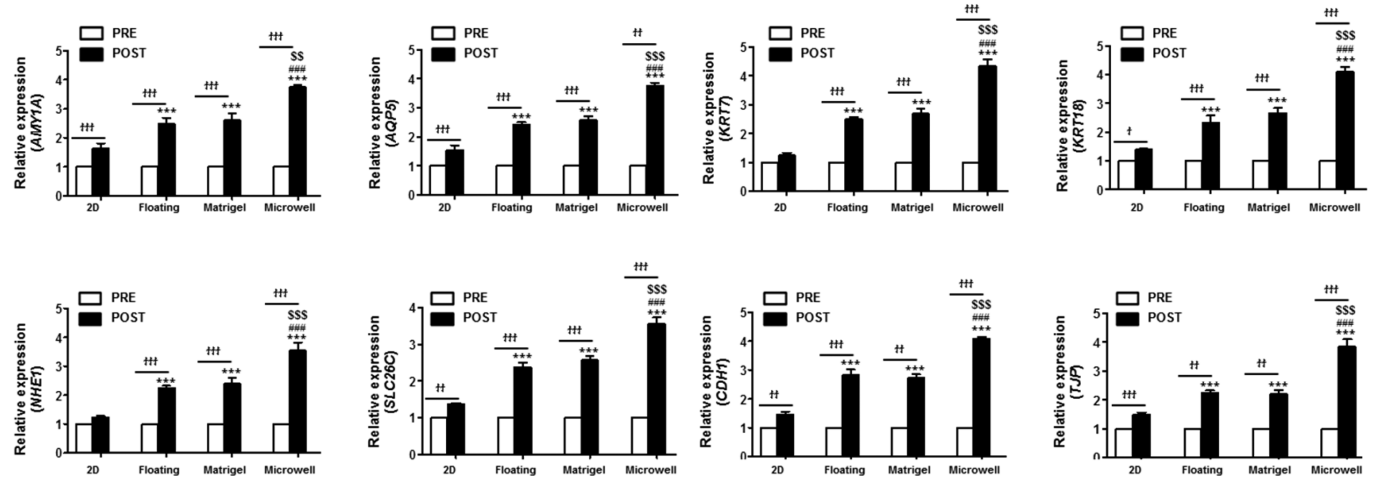

B
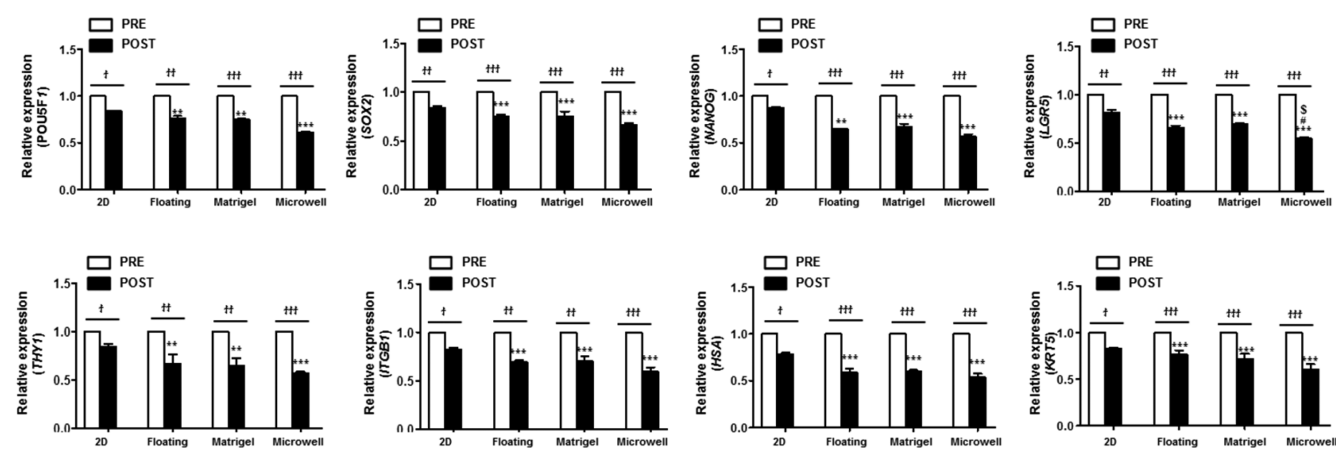

Figure 4. Salivary phenotypic marker and stem cell marker changes at the mRNA level. (A) mRNA levels of salivary acinar cell markers (AMY1A and $A Q P 5)$, salivary ductal cell markers (KRT7, KRT18, NHE1, and SLC26C), intercellular adherence protein (CDH1), and tight junction protein (TJP), as well as (B) stem cell-related markers (POUSF1, NANOG, LGR5, THY1, ITGB1, HAS, and KRT5) were tested by real-time PCR in 2D plastic and $3 \mathrm{D}$ spheroid at 3 days after salivary epithelial differentiation. The data from the three independent experiments were analyzed and presented as the mean \pm standard errors of the mean $(n=9)$. Two-way ANOVA, Bonferroni post hoc test. *, compared with POST in the 2D plastic group; \#, compared with POST the floating culture group; $\$$, compared with POST in the Matrigel group. $* P<0.05$; $\dagger$, compared between PRE and POST. $* * * P<0.001$, ${ }^{\# \# \# P} P<0.001,{ }^{\$} P<0.01,{ }^{\$ \$ \$} P<0.001,{ }^{\dagger} P<0.05,{ }^{\dagger} P<0.01,{ }^{\dagger \dagger} P<0.001$.

differentiation induction for 3 days (Figure 4A, B). RT-PCR revealed that the mRNA expression levels of salivary epithelial (acinar and ductal) cells were upregulated over time during the induction of initiation of lineage commitments in the Matrigel, floating culture, and microwell culture methods over 3 days, whereas the stem cell-related markers were downregulated during 3D organoid formation (Figure 4A, B). The relative changes in the expression of transcripts were more significant in the microwells, which suggested that the organization efficacy of single clonal SGSCs were enhanced in the microwells, in comparison with 2D, 3D floating, and Matrigel culture methods.

Protein translation was confirmed by Western blot, and the results showed that the corresponding changes in the expression of salivary epithelial ( $\alpha$-amylase, AQP5, E-cadherin, ZO-1, CK7, and CK18) or stem cell-related (OCT4, SOX2, NANOG, LGR5, CD90, and KRT5) markers were observed (Figure 5A, B). The relative levels of salivary epithelial proteins post- to preinduction significantly increased in the microwells relative to 2D, 3D floating, and Matrigel culture methods (Figure 5A). In contrast, stem cell-related markers markedly decreased in the microwells relative to the $2 \mathrm{D}, 3 \mathrm{D}$ floating, and Matrigel culture methods (Figure 5B). Collectively, the single clonal SGSCs can give rise to salivary epithelial cells that organize to form $3 \mathrm{D}$ organoids under 3D culture conditions with greatest the efficacy being observed in microwell culture.

3D Structures Organized from SGSCs Exhibit Functionally Robust Salivary Organoids. The 3D organoid cultures enabled us to evaluate the functionalities of assembled organoids after the induction of salivary epithelial differentiation from single clonal SGSCs. When we tested the activities of amylase released from the organoids after induction for 3 days, the mean levels of amylase activity, normalized to the number of cells, increased under the $3 \mathrm{D}$ organoid culture conditions, with the highest activity in microwells (Figure 6A). The level of $\alpha$ amylase in the medium was compared by Western blotting analysis and showed that the expression level of $\alpha$-amylase increased in the $3 \mathrm{D}$ organoid culture conditions and in the presence of adrenergic agonists (norepinephrine or isoproterenol). The excretion of $\alpha$-amylase increased more from cells cultured in the microwells relative to the floating or Matrigel culture methods (Figure 6B).

Next, we measured intracellular $\mathrm{pH}$ levels to assess ductal function $\left(\mathrm{Cl}^{-} / \mathrm{HCO}_{3}{ }^{-}\right.$exchange: $\mathrm{CBE}$ activity), in which $\mathrm{CBE}$ is essential for ductal $\mathrm{HCO}_{3}{ }^{-}$secretion in the salivary glands. After the addition of the $\mathrm{Cl}^{-} / \mathrm{HCO}_{3}{ }^{-}$medium, the intensities of $[\mathrm{pH}]_{\mathrm{i}}$ in the baseline $\left(\mathrm{Cl}^{-} /\right.$free $\left.\mathrm{HCO}_{3}{ }^{-}\right)$medium increased significantly, and greater increases were observed in the $3 \mathrm{D}$ organoids cultured in the microwells (Figure 6C).

Finally, we measured agonist-induced intracellular calcium concentrations $\left(\left[\mathrm{Ca}^{2+}\right]_{\mathrm{i}}\right)$ to test fluid secretory function. After the addition of calcium release agonists, carbachol (cholinergic agonist), ATP (purinergic receptor agonist), or thapsigargin (a potent inhibitor of the calcium-ATPase pump) to the culture medium, the intensities of $\left[\mathrm{Ca}^{2+}\right]_{\mathrm{i}}$ at the baseline increased 

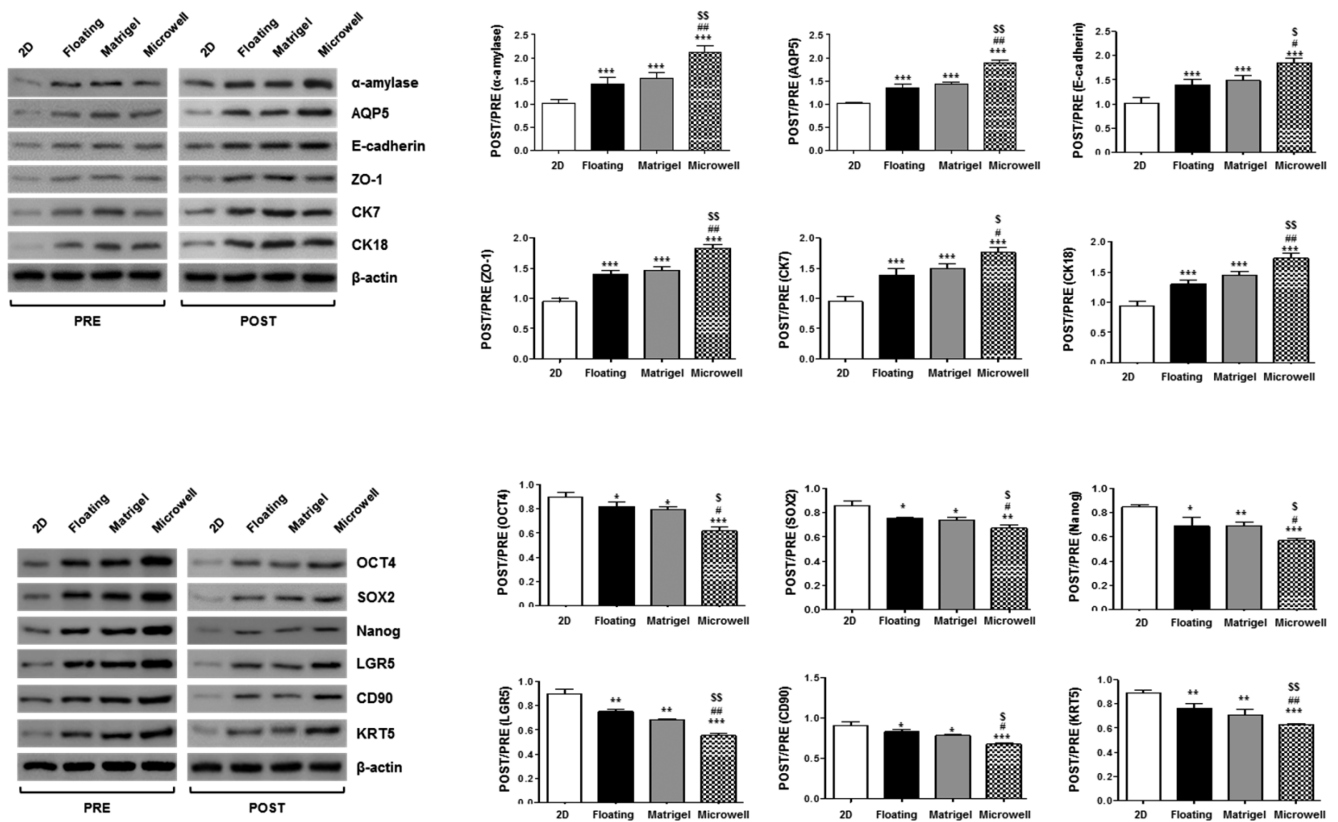

Figure 5. Salivary phenotypic marker and stem cell marker changes at the protein level after organization induction. The protein translation of the salivary epithelial ( $\alpha$-amylase, AQP5, E-cadherin, ZO-1, CK7, and CK18) or stem cell-related (OCT4, SOX2, NANOG, LGR5, CD90, and KRT5) markers was examined by Western blot, and the expression levels relative to $\beta$-actin were calculated. The data from three independent experiments were analyzed and presented as the mean \pm standard errors of the mean $(n=3)$. One-way ANOVA, Tukey's post hoc test. *, compared with $2 \mathrm{D}$ plastic; $\#$, compared with floating culture; $\$$, compared with Matrigel. ${ }^{* * *} P<0.001,{ }^{\#} P<0.05,{ }^{\# \#} P<0.01,{ }^{\# \# \#} P<0.001,{ }^{\$} P<0.05,{ }^{\$ \$} P<0.01$.

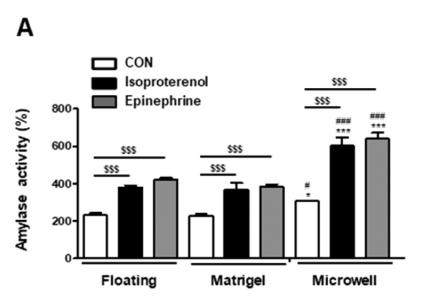

D

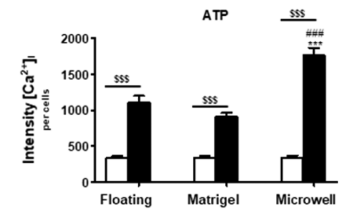

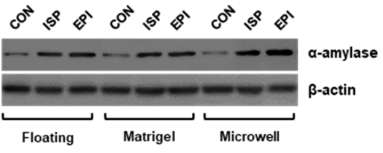

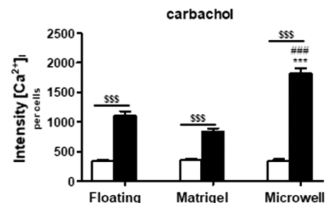

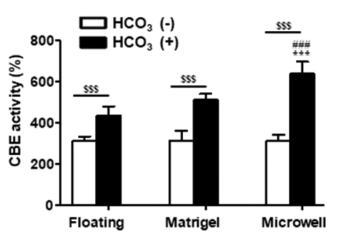

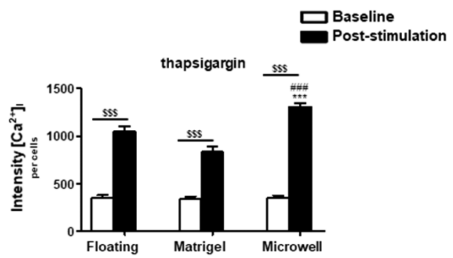

Figure 6. Promotion of salivary organoid functionality by 3D microwell cultures. (A) Effect of isoproterenol and epinephrine on amylase secretion in SGSCs. The amylase activities per wells containing $1 \times 10^{5}$ SGSCs were examined using an assay kit. The data are presented as means \pm standard errors of the mean. The data from three independent experiments were analyzed and presented as a mean \pm standard errors of the mean $(n=3)$. Two-way ANOVA, Bonferroni post hoc test. *, compared with the floating culture method; \#, compared with the Matrigel group; \$, compared with CON in each group. $* P<0.05, * * * P<0.001$, ${ }^{* \# \#} P<0.001,{ }^{\$ \$} P<0.001$. (B) Amylase expression in the cell supernatants was tested by Western blotting analysis in 2D plastic and 3D spheroid at 3 days. (C) The measurement of intracellular $\mathrm{pH}$ level. SGSCs were loaded with $\mathrm{BCECF}-\mathrm{AM}^{-}$and pretreated $\mathrm{Cl}^{-} /$free $\mathrm{HCO}_{3}^{-}$medium. After the addition of $\mathrm{Cl}^{-} / \mathrm{HCO}^{-}$medium, the intensities of $[\mathrm{pH}]$ in baseline $\left(\mathrm{Cl}^{-} /\right.$free $\left.\mathrm{HCO}_{3}^{-}\right)$medium increased significantly, with greater increases being observed in the 3D spheroids in microwells. The data from three independent experiments were analyzed and presented as the mean fluorescent intensity \pm standard errors of the mean $(n=3)$. Two-way ANOVA, Bonferroni post hoc test. *, compared with the floating group; \#, compared with the Matrigel group; $\$$, compared between $\mathrm{HCO}_{3}^{-}(-)$and $\mathrm{HCO}_{3}^{-}(+)$in each group. $* * * P<0.001,{ }^{\# \# \#} P<0.001,{ }^{\$ \$ \$} P<0.001$. $(\mathrm{C})$ Measurement of intracellular calcium level after stimulation with agonists. The hPECs were loaded with Fluo 4 and treated with adenosine triphosphate (ATP), carbachol, or thapsigargin for $30 \mathrm{~s}$ in the media. The data from three independent experiments were analyzed and presented as the mean fluorescent intensity \pm standard errors of the mean $(\mathrm{n}=3)$. Two-way ANOVA, Bonferroni post hoc test. *, compared with floating group; \#, compared with Matrigel group; \$, compared between the baseline and after-stimulation in each group. ${ }^{* * *} P<0.001,{ }^{\# \#} P<0.001,{ }^{\$ \$} P<0.001$.

significantly, and greater increases were observed in the $3 \mathrm{D}$ organoids cultured in the microwells (Figure 6D).

In Vivo Transplant of 3D Organoids Assembled from SGSCs in the Microwell Culture. We next tested whether the resultant $3 \mathrm{D}$ assembly can engraft and generate salivary tissue when transplanted into salivary glands. At 14 days after transplant, the organoids successfully engrafted into recipient salivary glands (Figure 7A). At 14 days post-transplant, histological examination revealed that hydrogel-encapsulated organoid cells grafted and preserved their structures with 
A

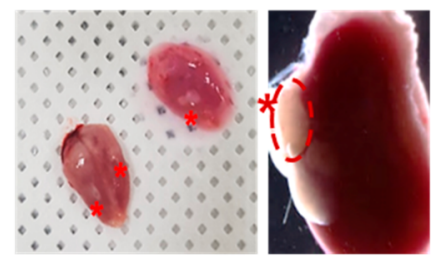

B

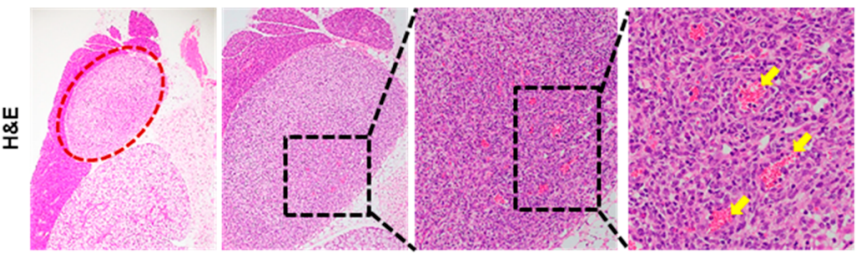

C

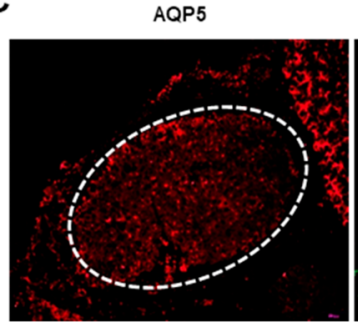

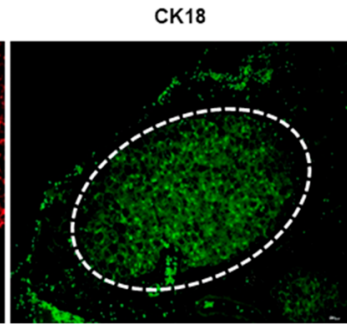
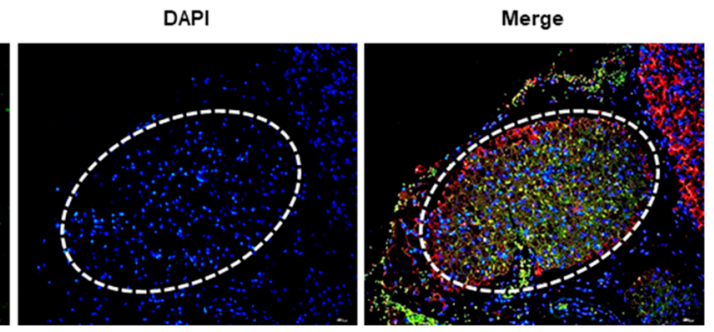

Figure 7. In vivo transplant of organoids assembled from SGSCs in the microwell culture. (A) Hydrogel-encapsulated organoids transplanted in vivo under submandibular capsule. (B) Representative histological pictures of H\&E staining at 14 days after transplantation. Arrows indicate capillaries in the neovascularature. Scale bars represent 400, 200, 100, and $20 \mu \mathrm{m}$. (C) Immunofluorescent staining for AQP5 and CK18 in transplanted organoid structures. Scale bars represent $200 \mu \mathrm{m}$.

neovascularization extending into transplanted areas (Figure $7 \mathrm{~B})$. Immunofluorcence staining revealed that the 3D organoids successfully engrafted in immunocompromised recipient mice salivary glands and maintained expression of acinar (AQP5) and ductal cell (CK18) markers (Figure 7C).

\section{DISCUSSION}

Traditionally, ASCs have been suspended and expanded in ECM-like 3D conditions for the rapid growth of epithelial tissues. The generation of "mini-gut" organoids from a single Lgr5 stem cell line has been established in Matrigel with several niche proteins, R-spondin (a Wnt signal amplifier and ligand of Lgr5), EGF, and the BMP inhibitor Noggin. ${ }^{8}$ Coppes and colleagues exploited floating sphere cells and Matrigel culture to obtain and expand salivary stem/progenitor cells, and they showed that it allowed the long-term expansion of salivary gland organoids in the presence of niche factors, such as Wnt3A and Rspondin, in order to control stem cell fate through activation of the Wnt pathway. ${ }^{15,16}$ In addition to the direct modulation of stem cells using niche factors, the indirect modulation using various $3 \mathrm{D}$ culture systems for recreation of the in vivo-mimetic extracellular microenvironment has been developed in many other bodies. In the system, the cells interacted with the ECM because of the topography, mechanical properties, or nonfibrous parts of ECM, such as adhesion molecules or growth factors.

In this study, we established a simple two-stage nicheindependent organization method from multipotent SGSCs based on microwell culture. We cultured SGSCs in microwells with standard DMEM, which enabled aggregation into spheroids to preactivate and prime the functions of the SGSCs, and then induced their differentiation and assembly into organoid structures that retain multicellular components by exchanging the medium to Hepato-STIM in the absence of niche factors. The results showed that we could prepare SGSCs with enhanced stemness by culture priming (spheroid), after which these primed SGSCs were used for the generation of more robust organoids upon differentiation induction by $3 \mathrm{D}$ organoid culture in microwells, compared with other traditional 3D culture methods, such as floating or Matrigel cultures. The resultant 3D organoids exhibited more prominent acinar and ductal markers, as well as better regulatory effects on acinar secretion and ductal $\mathrm{pH}$, than those from traditional 3D cultures.

In recent years, the culture of ASCs in $3 \mathrm{D}$, especially as spheroids, has been exploited to modulate their therapeutic potential, such as the promotion of stemness and/or the enhancement of paracrine functions. The priming approaches for the preactivation of stem cell functions prior to use of stem cells were enabled by the promotion of cell-to-cell or cell-toECM interactions by using 3D culture methods. In this study, we employed our array of microwells made by cell-repellent PEG hydrogel-micropattern incorporated-PCL nanofibrous scaffolds for 3D culture, and we could successfully obtain the primed SGSCs through the promotion of cell-to-cell and cell-to-ECM interactions. Indeed, in another recent study, we confirmed the enhanced therapeutic potential of 3D-primed SGSCs by 3D spheroid culture in microwells and their enhanced functions that were related to the activation of Wnt signaling. ${ }^{17}$ Although we used homogeneous clonal stem cells isolated by subfractionation methods, ${ }^{12}$ the efficiency of differentiation from our clonal SGSCs varied in accordance with the culture conditions, as well as clonal populations. Therefore, obtaining robust SGSCs that possess enhanced potency for differentiation into salivary cells appears to be beneficial for the optimization of proper organization conditions.

Along with multipotent tissue stem cells, a scaffold is another important factor to be considered for generation of organoids. As Lgr5-expressing ASCs have been used for the generation of $3 \mathrm{D}$ organoids, various $3 \mathrm{D}$ organoid culture systems by mimicking the in vivo niche environment have been established in mouse and human tissues, including the colon, stomach, liver, prostate, pancreas, ovaries, lingual mucosa, and taste buds. ${ }^{9,10,18-23}$ The generation of organoids from ASCs requires a defined set of niche factors and $3 \mathrm{D}$ culture using Matrigel. Although it has provided an innovative technology to generate a self-organizing $3 \mathrm{D}$ structure that mimics in vivo architecture, functionality, and genetic signature of the original tissues, 3D culture uses animal-derived matrices (Matrigel and its relative basement membrane extract). Recently, traditional 3D culture 
methods, such as floating or hanging drop culture, have been employed for generation of stem-cell-driven 3D organization. ${ }^{16,24}$ Synthetic agarose gel-replicated organotypic scaffolds or natural ECM scaffolds were also introduced for assembly of salivary-gland microtissue. ${ }^{25,26}$

SGSC can be cultured using synthetic or natural scaffolds which have been widely used in various tissue engineering applications. We chose synthetic PCL fibers as main scaffolds. Synthetic polymers have advantages over natural polymer since their material properties are typically more flexible and controllable than those of natural materials. Although synthetic polymeric scaffolds lack the biological cues inherent in natural scaffolds including decellularized scaffolds and natural extracellular matrix scaffolds, they can be easily available, nontoxic, inexpensive, biocompatible, and tuned to promote desirable biological responses. ${ }^{27}$ PEG hydrogel micropatterns were incorporated into PCL fibrous scaffolds, which would facilitate the formation of spheroids with uniform size.

We previously established a method for 3D organization of salivary-gland-related cells by culturing hPECs in the nanoscaffold microwell system. ${ }^{13}$ However, we thought that this 3D culture of hPECs might not be sufficient for bioengineering of an artificial biosalivary gland and that it is still necessary to develop more complex organlike structures with a combination of various cell components, as well as neural and/or vascular networks. Because we successfully isolated single-clone SGSCs by a subfractionation culturing method, in this study, we were able to induce salivary organization from multipotent SGSCs without exogenous niche factors under 3D culture conditions. Furthermore, SGSC-driven organoids could be generated in a regular size and controllable fashion in the microwells, and this technology can be applied as a high-throughput platform for organotypic microarray-driven organization from ASCs.

Nevertheless, our niche-independent organization method has limitations, because it is based on the induction of differentiation from multipotent tissue-resident ASCs. Although SGSCs can produce both epithelial and mesenchymal lineage cells, the organizing structures do not exhibit in vivo-like acinoductal structures with microenvironments. In this study, we used single clonal SGSCs and a subfractionation culturing method that may be sufficient for the isolation and production of homogeneous clonal tissue-resident ASCs, as we have previously reported in different sources of tissue-resident stem cells. ${ }^{11,12,28}$ However, the existence of different ASC populations with nonidentical characteristics has been reported, even in the same batch of ASCs, which is indicative of the cellular heterogeneity of ASCs and the technical limitations of single stem cell-based organization strategies. Therefore, efforts should be made to improve the ASCs-based organization efficacy in 3D culture matrices with or without niche factors.

Recently, it was proposed that ASCs can be used not only for cell therapeutics, but also for the replacement of hypofunctional tissue or organs by transplants of organ-like structures. ${ }^{29}$ Although SG-resident stem/progenitor cells possess a multipotent potential to give rise to various SG cells, inducing organization from naive SG stem/progenitor cells has not yet been explored. From this perspective, our novel culture method for the generation of salivary organoids can be used as a platform for organoid transplants. Furthermore, our microwell-driven organization method is based on the assembly of homogeneous single clonal stem cells under xeno-free (Matrigel-free) culture conditions. To our best knowledge, this is the first research to demonstrate that SGSCs can generate salivary organoids in niche factor-independent 3D culture conditions. This study may be a prerequisite step for future preclinical and clinical studies aimed at organoid-based tissue engineering and regeneration. Further investigation is needed to investigate the regenerative potentials and mechanisms of $3 \mathrm{D}$ culture-driven organoids in vivo. In addition, it would be valuable to determine the applicability of this culture method to disease modeling, drug testing, or genetic editing from patient-derived organoids.

\section{CONCLUSIONS}

We established a protocol for single clonal SGSC-derived organization using niche-independent $3 \mathrm{D}$ microwell culture. Through two simple steps, priming and differentiation, we could obtain salivary organoids phenotypically and functionally relevant to salivary gland epithelial tissues. Moreover, our organization methods showed better efficiency in 3D organization in terms of structure and function than traditional 3D culture methods, such as Matrigel embedding or floating cultures. Although this novel niche-independent organization method enabled xeno-free, defined, and biomimetic differentiation, further investigations are needed to analyze the selforganizing capacity by the addition of niche factors and to evaluate the applications of organoids generated from single clonal SGSCs.

\section{ASSOCIATED CONTENT}

\section{Supporting Information}

The Supporting Information is available free of charge on the ACS Publications website at DOI: 10.1021/acsbiomaterials.8b00894.

Tables S1-S4 providing more detailed information about the materials used in the experiments (PDF)

\section{AUTHOR INFORMATION}

\section{Corresponding Authors}

*Email: wongun@yonsei.ac.kr. Tel: +82-2-2123-5755. Fax: +82-2-312-6401 (W.-G.K.).

*Email: jylimmd@yuhs.ac. Tel: 82-2-2019-3460. Fax: 82-23463-4750 (J.-Y.L.).

ORCID $\odot$

Won-Gun Koh: 0000-0002-5191-2531

Jae-Yol Lim: 0000-0002-9757-6414

\section{Author Contributions}

${ }^{\S}$ W.-G.K. and J.-Y.L. contributed equally to this work.

\section{Notes}

The authors declare no competing financial interest.

\section{ACKNOWLEDGMENTS}

This work was supported by the Stem Cell Research Project through the National Research Foundation of Korea (NRF) funded by the Ministry of Education, Science and Technology (NRF-2017M3A9B4032053) and by the Basic Science Research Program through the National Research Foundation of Korea (NRF) funded by the Ministry of Science, ICT and Future Planning (2018M3A9E2024583), Republic of Korea.

\section{REFERENCES}

(1) Shamir, E. R.; Ewald, A. J. Three-Dimensional Organotypic Culture: Experimental Models of Mammalian Biology and Disease. Nat. Rev. Mol. Cell Biol. 2014, 15 (10), 647-664. 
(2) Yin, X.; Mead, B. E.; Safaee, H.; Langer, R.; Karp, J. M.; Levy, O. Engineering Stem Cell Organoids. Cell Stem Cell 2016, 18 (1), 25-38.

(3) Clevers, H. Modeling Development and Disease with Organoids. Cell 2016, 165 (7), 1586-1597.

(4) Eiraku, M.; Takata, N.; Ishibashi, H.; Kawada, M.; Sakakura, E.; Okuda, S.; Sekiguchi, K.; Adachi, T.; Sasai, Y. Self-Organizing OpticCup Morphogenesis in Three-Dimensional Culture. Nature 2011, 472 (7341), 51-56.

(5) Lancaster, M. A.; Renner, M.; Martin, C. A.; Wenzel, D.; Bicknell, L. S.; Hurles, M. E.; Homfray, T.; Penninger, J. M.; Jackson, A. P.; Knoblich, J. A. Cerebral Organoids Model Human Brain Development and Microcephaly. Nature 2013, 501 (7467), 373-379.

(6) Spence, J. R.; Mayhew, C. N.; Rankin, S. A.; Kuhar, M. F.; Vallance, J. E.; Tolle, K.; Hoskins, E. E.; Kalinichenko, V. V.; Wells, S. I.; Zorn, A. M.; Shroyer, N. F.; Wells, J. M. Directed Differentiation of Human Pluripotent Stem Cells into Intestinal Tissue in Vitro. Nature 2011, 470 (7332), 105-109.

(7) Takebe, T.; Sekine, K.; Enomura, M.; Koike, H.; Kimura, M.; Ogaeri, T.; Zhang, R. R.; Ueno, Y.; Zheng, Y. W.; Koike, N.; Aoyama, S.; Adachi, Y.; Taniguchi, H. Vascularized and Functional Human Liver from an Ipsc-Derived Organ Bud Transplant. Nature 2013, 499 (7459), 481-484.

(8) Sato, T.; Vries, R. G.; Snippert, H. J.; van de Wetering, M.; Barker, N.; Stange, D. E.; van Es, J. H.; Abo, A.; Kujala, P.; Peters, P. J.; Clevers, H. Single Lgr5 Stem Cells Build Crypt-Villus Structures in Vitro without a Mesenchymal Niche. Nature 2009, 459 (7244), 262-265.

(9) Barker, N.; Huch, M.; Kujala, P.; van de Wetering, M.; Snippert, H. J.; van Es, J. H.; Sato, T.; Stange, D. E.; Begthel, H.; van den Born, M.; Danenberg, E.; van den Brink, S.; Korving, J.; Abo, A.; Peters, P. J.; Wright, N.; Poulsom, R.; Clevers, H. Lgr5 $(+\mathrm{Ve})$ Stem Cells Drive SelfRenewal in the Stomach and Build Long-Lived Gastric Units in Vitro. Cell Stem Cell 2010, 6 (1), 25-36.

(10) Huch, M.; Bonfanti, P.; Boj, S. F.; Sato, T.; Loomans, C. J.; van de Wetering, M.; Sojoodi, M.; Li, V. S.; Schuijers, J.; Gracanin, A.; Ringnalda, F.; Begthel, H.; Hamer, K.; Mulder, J.; van Es, J. H.; de Koning, E.; Vries, R. G.; Heimberg, H.; Clevers, H. Unlimited in Vitro Expansion of Adult Bi-Potent Pancreas Progenitors through the Lgr5/ R-Spondin Axis. EMBO J. 2013, 32 (20), 2708-2721.

(11) Lim, J. Y.; Yi, T.; Lee, S.; Kim, J.; Kim, S. N.; Song, S. U.; Kim, Y. M. Establishment and Characterization of Mesenchymal Stem CellLike Clonal Stem Cells from Mouse Salivary Glands. Tissue Eng., Part C 2015, 21 (5), 447-457.

(12) Yi, T.; Lee, S.; Choi, N.; Shin, H. S.; Kim, J.; Lim, J. Y. Single Cell Clones Purified from Human Parotid Glands Display Features of Multipotent Epitheliomesenchymal Stem Cells. Sci. Rep. 2016, 6, 36303.

(13) Shin, H. S.; Kook, Y. M.; Hong, H. J.; Kim, Y. M.; Koh, W. G.; Lim, J. Y. Functional Spheroid Organization of Human Salivary Gland Cells Cultured on Hydrogel-Micropatterned Nanofibrous Microwells. Acta Biomater. 2016, 45, 121-132.

(14) Lee, H. J.; Koh, W. G. Hydrogel Micropattern-Incorporated Fibrous Scaffolds Capable of Sequential Growth Factor Delivery for Enhanced Osteogenesis of Hmscs. ACS Appl. Mater. Interfaces 2014, 6 (12), 9338-9348.

(15) Nanduri, L. S.; Baanstra, M.; Faber, H.; Rocchi, C.; Zwart, E.; de Haan, G.; van Os, R.; Coppes, R. P. Purification and Ex Vivo Expansion of Fully Functional Salivary Gland Stem Cells. Stem Cell Rep. 2014, 3 (6), 957-964

(16) Maimets, M.; Rocchi, C.; Bron, R.; Pringle, S.; Kuipers, J.; Giepmans, B. N.; Vries, R. G.; Clevers, H.; de Haan, G.; van Os, R.; Coppes, R. P. Long-Term in Vitro Expansion of Salivary Gland Stem Cells Driven by Wnt Signals. Stem Cell Rep. 2016, 6 (1), 150-162.

(17) Shin, H. S.; Lee, S.; Hong, H. J.; Lim, Y. C.; Koh, W. G.; Lim, J. Y. Stem Cell Properties of Human Clonal Salivary Gland Stem Cells Are Enhanced by Three-Dimensional Priming Culture in Nanofibrous Microwells. Stem Cell Res. Ther. 2018, 9 (1), 74

(18) Sato, T.; Stange, D. E.; Ferrante, M.; Vries, R. G.; Van Es, J. H.; Van den Brink, S.; Van Houdt, W. J.; Pronk, A.; Van Gorp, J.; Siersema, P. D.; Clevers, H. Long-Term Expansion of Epithelial Organoids from
Human Colon, Adenoma, Adenocarcinoma, and Barrett's Epithelium. Gastroenterology 2011, 141 (5), 1762-1772.

(19) Huch, M.; Dorrell, C.; Boj, S. F.; van Es, J. H.; Li, V. S.; van de Wetering, M.; Sato, T.; Hamer, K.; Sasaki, N.; Finegold, M. J.; Haft, A.; Vries, R. G.; Grompe, M.; Clevers, H. In Vitro Expansion of Single Lgr5+ Liver Stem Cells Induced by Wnt-Driven Regeneration. Nature 2013, 494 (7436), 247-250.

(20) Karthaus, W. R.; Iaquinta, P. J.; Drost, J.; Gracanin, A.; van Boxtel, R.; Wongvipat, J.; Dowling, C. M.; Gao, D.; Begthel, H.; Sachs, N.; Vries, R. G.; Cuppen, E.; Chen, Y.; Sawyers, C. L.; Clevers, H. C. Identification of Multipotent Luminal Progenitor Cells in Human Prostate Organoid Cultures. Cell 2014, 159 (1), 163-175.

(21) Kessler, M.; Hoffmann, K.; Brinkmann, V.; Thieck, O.; Jackisch, S.; Toelle, B.; Berger, H.; Mollenkopf, H. J.; Mangler, M.; Sehouli, J.; Fotopoulou, C.; Meyer, T. F. The Notch and Wnt Pathways Regulate Stemness and Differentiation in Human Fallopian Tube Organoids. Nat. Commun. 2015, 6, 8989.

(22) Hisha, H.; Tanaka, T.; Kanno, S.; Tokuyama, Y.; Komai, Y.; Ohe, S.; Yanai, H.; Omachi, T.; Ueno, H. Establishment of a Novel Lingual Organoid Culture System: Generation of Organoids Having Mature Keratinized Epithelium from Adult Epithelial Stem Cells. Sci. Rep. 2013, 3, 3224.

(23) Ren, W.; Lewandowski, B. C.; Watson, J.; Aihara, E.; Iwatsuki, K.; Bachmanov, A. A.; Margolskee, R. F.; Jiang, P. Single Lgr5- or Lgr6Expressing Taste Stem/Progenitor Cells Generate Taste Bud Cells Ex Vivo. Proc. Natl. Acad. Sci. U. S. A. 2014, 111 (46), 16401-16406.

(24) Becavin, T.; Kuchler-Bopp, S.; Kokten, T.; Huck, O.; Messaddeq, N.; Lesot, H.; Deveaux, E.; Benkirane-Jessel, N.; Laetitia, K. WellOrganized Spheroids as a New Platform to Examine Cell Interaction and Behaviour During Organ Development. Cell Tissue Res. 2016, 366 (3), 601-615.

(25) Ozdemir, T.; Srinivasan, P. P.; Zakheim, D. R.; Harrington, D. A.; Witt, R. L.; Farach-Carson, M. C.; Jia, X.; Pradhan-Bhatt, S. Bottom-up Assembly of Salivary Gland Microtissues for Assessing Myoepithelial Cell Function. Biomaterials 2017, 142, 124-135.

(26) Lilliu, M. A.; Seo, Y. J.; Isola, M.; Charbonneau, A. M.; Zeitouni, A.; El-Hakim, M.; Tran, S. D. Natural Extracellular Matrix Scaffolds Recycled from Human Salivary Digests: A Morphometric Study. Oral Dis 2016, 22 (4), 313-323.

(27) Place, E. S.; George, J. H.; Williams, C. K.; Stevens, M. M. Synthetic Polymer Scaffolds for Tissue Engineering. Chem. Soc. Rev. 2009, 38 (4), 1139-1151.

(28) Yi, T.; Kim, S. N.; Lee, H. J.; Kim, J.; Cho, Y. K.; Shin, D. H.; Tak, S. J.; Moon, S. H.; Kang, J. E.; Ji, I. M.; Lim, H. J.; Lee, D. S.; Jeon, M. S.; Song, S. U. Manufacture of Clinical-Grade Human Clonal Mesenchymal Stem Cell Products from Single Colony Forming Unit-Derived Colonies Based on the Subfractionation Culturing Method. Tissue Eng., Part C 2015, 21 (12), 1251-1262.

(29) Ogawa, M.; Oshima, M.; Imamura, A.; Sekine, Y.; Ishida, K.; Yamashita, K.; Nakajima, K.; Hirayama, M.; Tachikawa, T.; Tsuji, T. Functional Salivary Gland Regeneration by Transplantation of a Bioengineered Organ Germ. Nat. Commun. 2013, 4, 2498. 